\title{
Control strategy on the double-diffusive convection in a nanofluid layer with internal heat generation
}

\begin{abstract}
The influences of feedback control and internal heat source on the onset of Rayleigh-Bénard convection in a horizontal nanofluid layer is studied analytically due to Soret and Dufour parameters. The confining boundaries of the nanofluid layer (bottom boundary-top boundary) are assumed to be free-free, rigid-free, and rigid-rigid, with a source of heat from below. Linear stability theory is applied, and the eigenvalue solution is obtained numerically using the Galerkin technique. Focusing on the stationary convection, it is shown that there is a positive thermal resistance in the presence of feedback control on the onset of doublediffusive convection, while there is a positive thermal efficiency in the existence of internal heat generation. The possibilities of suppress or augment of the Rayleigh-Bénard convection in a nanofluid layer are also discussed in detail.
\end{abstract}

